\title{
A Study of the Strong Gravity Region of the Black Hole in GS 1354-645
}

\author{
Yerong $\mathrm{Xu}^{1}$, Sourabh Nampalliwar ${ }^{2}$, Askar B. Abdikamalov ${ }^{1}$, Dimitry Ayzenberg ${ }^{1}$, \\ Cosimo Bambi ${ }^{1,2}$ (D), Thomas Dauser ${ }^{3}$, Javier A. García ${ }^{3,4}$, and Jiachen Jiang ${ }^{5}$ \\ ${ }^{1}$ Center for Field Theory and Particle Physics and Department of Physics, Fudan University, 200438 Shanghai, People's Republic of China; bambi@fudan.edu.cn \\ ${ }^{2}$ Theoretical Astrophysics, Eberhard-Karls Universität Tübingen, D-72076 Tübingen, Germany \\ ${ }^{3}$ Remeis Observatory \& ECAP, Universität Erlangen-Nürnberg, D-96049 Bamberg, Germany \\ ${ }^{4}$ Cahill Center for Astronomy and Astrophysics, California Institute of Technology, Pasadena, CA 91125, USA \\ ${ }^{5}$ Institute of Astronomy, University of Cambridge, Cambridge CB3 OHA, UK \\ Received 2018 July 27; revised 2018 August 14; accepted 2018 August 18; published 2018 October 1
}

\begin{abstract}
It is thought that the spacetime metric around astrophysical black holes is well described by the Kerr solution of Einstein's gravity. However, robust observational evidence of the Kerr nature of these objects is still lacking. Here we fit the X-ray spectrum of the stellar-mass black hole in GS 1354-645 with a disk reflection model beyond Einstein's gravity in order test the Kerr black hole hypothesis. We consider the Johannsen metric with the deformation parameters $\alpha_{13}$ and $\alpha_{22}$. The Kerr metric is recovered for $\alpha_{13}=\alpha_{22}=0$. For $\alpha_{22}=0$, our measurements of the black hole spin and of the deformation parameter $\alpha_{13}$ are $a_{*}>0.975$ and $-0.34<\alpha_{13}<0.16$, respectively. For $\alpha_{13}=0$, we find $a_{*}>0.975$ and $-0.09<\alpha_{22}<0.42$. All the reported uncertainties are at $99 \%$ of confidence level for two relevant parameters.
\end{abstract}

Key words: accretion, accretion disks - black hole physics - gravitation

\section{Introduction}

Einstein's theory of general relativity (GR hereafter) is the standard model for describing the effects of gravity in our universe today. Predictions of GR range from corrections at the scale of Earth (Williams et al. 2004; Everitt et al. 2011) and solar system (Bertotti et al. 2003) to entirely new horizons (Kramer et al. 2006; Abbott et al. 2016). While the theory has been largely successful in explaining the observations, lingering issues (Sahni 2004; Frieman et al. 2008) motivate us to look beyond. Besides observations, there are theoretical reasons to expect a future theory that will supersede GR (Mathur 2005; Dvali \& Gomez 2013; Giddings 2014, 2017; Berti et al. 2015). Thus, determining the domain of validity of GR is important from both observational and theoretical reasons. Many alternative theories have been proposed in this regard. Since GR has been rigorously tested in the weak-field regime, these alternative theories have the same predictions as GR for in this regime and present deviations only when gravity becomes strong.

Black holes are an important prediction of GR. They are expected to be formed in the core collapse of massive stars, and as the final product of mergers of massive objects. Other avenues include supermassive black holes, which reside at center of galaxies, and intermediate mass black holes, which are possibly present in globular clusters. Being extremely compact (i.e., the ratio $M / R$ being close to 1 ), black holes are the ideal candidates for testing GR in the so-called strong-field regime (Yunes \& Siemens 2013; Bambi et al. 2016; Yagi \& Stein 2016; Bambi 2017).

In four-dimensional GR, uncharged black holes are completely described by two parameters, which are the mass, $M$, and the spin angular momentum, $J$, of the object. This is the well-known result of the no-hair theorem, which holds under specific assumptions (Carter 1971; Robinson 1975). There is also a uniqueness theorem, asserting that the only uncharged black hole solution in four-dimensional Einstein's gravity is the Kerr metric. One approach then to test GR is to check whether the spacetime metric around astrophysical black holes is indeed described by the Kerr geometry (Bambi 2012, 2013; Krawczynski 2012; Johannsen \& Psaltis 2013; Jiang et al. 2015). ${ }^{6}$

We have developed a framework recently to look for deviations away from the Kerr metric using X-ray reflection spectroscopy (Bambi et al. 2017, 2018). The astrophysical system consists of a compact object that is described by a parametrically deformed Kerr metric (quantized by deformation parameters) with an accretion disk and a corona. In Bambi et al. (2017, 2018) we introduce the model we developed for use in Xspec (Arnaud 1996), which is the standard software for X-ray data analysis. In Cao et al. (2018), we presented the first constraints on one of the deformation parameters introduced in Johannsen (2013) using observations of the narrow line Seyfert 1 galaxy 1H0707-495. Constraints using another narrow line Seyfert 1 galaxy, Ark 564, were reported in Tripathi et al. (2018). And in Wang-Ji et al. (2018), we report constraints using the X-ray binary GX 339-4. The current paper is an analysis in this series. Here we present individual constraints on two parameters of Johannsen (2013) using observations of the lowmass X-ray binary GS 1354-645. We also discuss some issues of the astrophysical modeling that arise naturally in such an analysis.

The outline of the paper is as follows. We review the nonKerr metric employed in this work and the astrophysical model in Section 2. Section 3 describes the source, the observation, and data reduction. Spectral analysis and results of the fitting are reported in Section 4. Results and some systematic issues are discussed in Section 5. We conclude in Section 6. Throughout the paper, we adopt the convention $c=G_{\mathrm{N}}=1$.

\section{Review}

Here we review only the most important aspects. More details on all of the aspects can be found in Bambi et al. (2017, 2018).

The metric we explore is an extension of the Kerr metric, proposed in Johannsen (2013). We employ such a metric

\footnotetext{
6 This approach has the limitation that if the spacetime is indeed described by the Kerr metric, we cannot differentiate between GR and alternative theories, since most alternative theories include the Kerr metric as a solution (Barausse \& Sotiriou 2008; Psaltis et al. 2008).
} 
because it has some interesting properties with respect to other parametric black hole spacetimes proposed in the literature. It is everywhere regular outside of the event horizon. It has three independent constants of motion, and thus admits first-order equations of motion as in the Kerr spacetime. As shown in Johannsen (2013), the metric can reproduce some known black hole solutions in modified theories of gravity (EinsteinDilaton-Gauss-Bonnet gravity, Chern-Simons gravity, and braneworld models) for specific choices of the values of its deformation parameters.

In Boyer-Lindquist coordinates, the line element of the Johannsen metric reads (we use units in which $G_{\mathrm{N}}=c=1$; Johannsen 2013)

$$
\begin{aligned}
d s^{2}= & -\frac{\tilde{\Sigma}\left(\Delta-a^{2} A_{2}^{2} \sin ^{2} \theta\right)}{B^{2}} d t^{2} \\
& -\frac{2 a\left[\left(r^{2}+a^{2}\right) A_{1} A_{2}-\Delta\right] \tilde{\Sigma} \sin ^{2} \theta}{B^{2}} d t d \phi \\
& +\frac{\tilde{\Sigma}}{\Delta A_{5}} d r^{2}+\tilde{\Sigma} d \theta^{2} \\
& +\frac{\left[\left(r^{2}+a^{2}\right)^{2} A_{1}^{2}-a^{2} \Delta \sin ^{2} \theta\right] \tilde{\Sigma} \sin ^{2} \theta}{B^{2}} d \phi^{2},
\end{aligned}
$$

where

$$
\begin{aligned}
a & =J / M, \quad \tilde{\Sigma}=\Sigma+f, \quad \Sigma=r^{2}+a^{2} \cos ^{2} \theta, \\
\Delta & =r^{2}-2 M r+a^{2}, \\
B & =\left(r^{2}+a^{2}\right) A_{1}-a^{2} A_{2} \sin ^{2} \theta,
\end{aligned}
$$

and

$$
\begin{aligned}
f & =\sum_{n=3}^{\infty} \epsilon_{n} \frac{M^{n}}{r^{n-2}}, \quad A_{1}=1+\sum_{n=3}^{\infty} \alpha_{1 n}\left(\frac{M}{r}\right)^{n}, \\
A_{2} & =1+\sum_{n=2}^{\infty} \alpha_{2 n}\left(\frac{M}{r}\right)^{n}, \\
A_{5} & =1+\sum_{n=2}^{\infty} \alpha_{5 n}\left(\frac{M}{r}\right)^{n} .
\end{aligned}
$$

We restrict our attention to the first-order deformation parameters $\alpha_{13}$ and $\alpha_{22}{ }^{7}$ The Kerr metric is recovered when $\alpha_{13}=\alpha_{22}=0$. In the Kerr metric, black holes only exist for $\left|a_{*}\right| \leqslant 1$, where $a_{*}=a / M=J / M^{2}$ is the dimensionless spin parameter. In the Johannsen spacetime, we still have the condition $\left|a_{*}\right| \leqslant 1$ for the existence of the event horizon. In order to exclude a violation of Lorentzian signature in the metric and the existence of closed time-like curves in the exterior region, we have to impose (Johannsen 2013)

$$
\begin{aligned}
& \alpha_{13}>-\left(1+\sqrt{1-a_{*}^{2}}\right)^{3}, \\
& \alpha_{22}>-\left(1+\sqrt{1-a_{*}^{2}}\right)^{2} .
\end{aligned}
$$

Additionally, the metric is singular for $B=0$, and thus we have to require that $B$ never vanishes outside of the event horizon (Tripathi et al. 2018). For $\alpha_{22}=0$, we have the

\footnotetext{
7 These two parameters have the strongest effect on the iron line (Bambi et al. 2017), so we restrict our analysis to these parameters.
}

constraint

$$
\alpha_{13}>-\frac{1}{2}\left(1+\sqrt{1-a_{*}^{2}}\right)^{4} .
$$

For $\alpha_{13}=0$, the constraint is

$$
\alpha_{22}<\frac{\left(1+\sqrt{1-a_{*}^{2}}\right)^{4}}{a_{*}^{2}} .
$$

Our exploration of the spin and the deformation parameters happens within these constraints.

The astrophysical system is modeled by a black hole surrounded by a disk and corona (Bambi 2018). The disk is modeled as a Novikov-Thorne-type geometrically thin, optically thick disk. The corona is described by a broken power-law spectra.

RELXILL_NK, an extension of RELXILL (Dauser et al. 2013; García et al. 2014), models the X-ray reflection spectrum of black holes described by the Johannsen metric in the disk-corona scenario. It uses the transfer function approach, introduced in Cunningham (1975) and described in Bambi et al. (2017). During each analysis, we allow one of the deformation parameters (i.e., one of $\alpha_{13}$ and $\alpha_{22}$ ) to vary, setting all of the others at zero.

\section{Observations and Data Reduction}

GS 1354-645, alias BW Cir, is a low-mass X-ray binary comprising of a black hole and a low-mass stellar companion. Using optical spectroscopy, the masses are dynamically confirmed at $M_{\mathrm{BH}} \geqslant 7.6 \pm 0.7 M_{\odot}$ and $M_{\mathrm{c}} \leqslant 1.2 M_{\odot}$ (Casares et al. 2009). The source was discovered in its 1987 outburst by the Japanese X-ray mission Ginga (Makino 1987). This was followed by another outburst in 1997, detected by RXTE (Brocksopp et al. 2001). A third outburst was detected in 2015 June by Swift/ BAT (Miller et al. 2015).

During the 2015 outburst, NuSTAR observed GS 1354-645 for three sessions: on June 13 (hereafter Obs 1) for about $24 \mathrm{ks}$, on July 11 (hereafter Obs 2) for about $30 \mathrm{ks}$, and on August 6 (hereafter Obs 3 ) for about $35 \mathrm{ks}$. The first two observations are studied in El-Batal et al. (2016). They found a truncated inner edge for the accretion disk at $700_{-500}^{+200} R_{\mathrm{ISCO}}$ for Obs 1 , while Obs 2 gave a tightly constrained inner edge, close to $R_{\mathrm{ISCO}}$. No analysis of Obs 3 has been reported yet.

In our analysis, we employed Xspec v12.9.1 (Arnaud 1996). We processed the data from both the focal plane module A (FPMA) and focal plane module B (FPMB) instruments using nupipeline $\mathrm{v} 0.4 .5$ with the standard filtering criteria and the NuSTAR CALDB version 20171002. We used the nuproducts routine to extract source spectra, responses, and background spectra. For the source, we chose a circular region with a radius of 148 arcsec. For the background, we chose a circular region with a radius of 148 arcsec on the same chip. All spectra were binned to a minimum of 30 counts before analysis to ensure the validity of the $\chi^{2}$ fit statistics. In what follows, we report the analysis of Obs 2 only. For Obs 1, we find that the inner edge of the accretion disk is truncated at large radii, in agreement with what was found in El-Batal et al. (2016) and making it unsuitable to constrain the background metric.

\section{Spectral Analysis}

We fit Obs 2 with the following models:

Model 1: TBABS*POWERLAW.

This is the simplest model. TBABS describes the Galactic absorption (Wilms et al. 2000) and we fix the 
Table 1

Summary of the Best-fit Values for Model 1 (Simple Power Law), Model 2a (Power Law with Reflection Component and $\alpha_{13}$ Free), Model 2b (as Model 2a but with $R_{\text {in }}$ Free), Model 2c (as Model 2a but with $q_{\text {out }}=3$ Frozen), and Model 3 (as Model 2a but with the Deformation Parameters $\alpha_{13}=0$ and $\alpha_{22}$ Free)

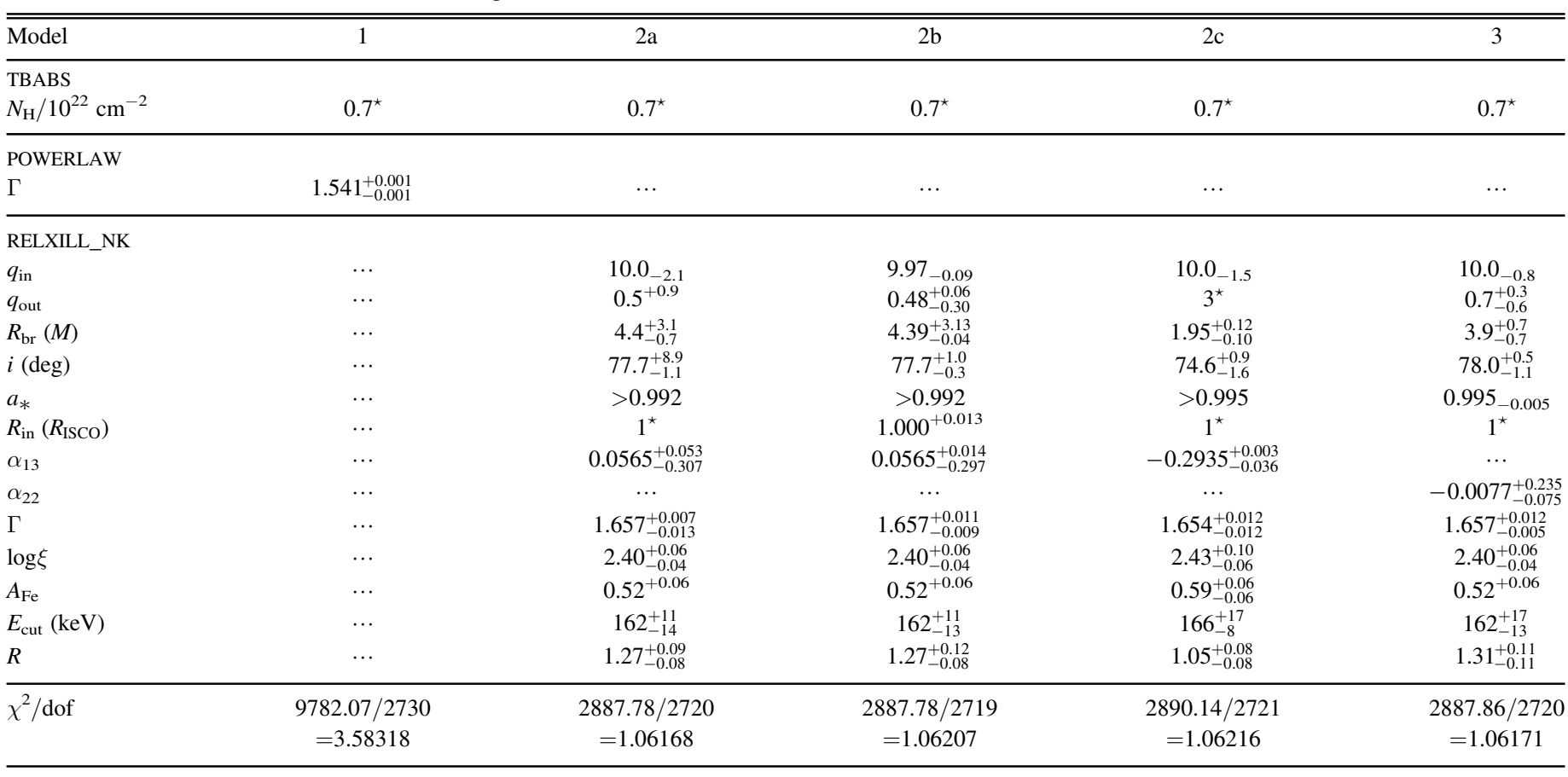

Note. The reported uncertainties correspond to the $90 \%$ confidence level for one relevant parameter. ${ }^{\star}$ indicates that the parameter is frozen. See the text for more details.

galactic column density to $N_{\mathrm{H}}=0.7 \times 10^{22} \mathrm{~cm}^{-2}$, which we obtain from the HEASARC column density tool, based on Dickey \& Lockman (1990). POWERLAW describes the power-law spectrum from the hot corona around the black hole. The best-fit values are reported in the second column in Table 1, where we see that the minimum of the reduced $\chi^{2}$ is around 3.6. Figure 1 (top panel) shows the data to the best-fit model ratio, and we clearly see a broad iron line at 6-7 keV and a Compton hump at $10-30 \mathrm{keV}$.

Model 2a: TBABS*RELXILL_NK with $\alpha_{13}$ as the deformation parameter.

We now use our model RELXILL_NK. The emissivity profile of the disk is modeled with a broken power law and is described by three free parameters: the inner emissivity index $q_{\text {in }}$, the outer emissivity index $q_{\text {out }}$, and the breaking radius $R_{\mathrm{br}}$. The inner edge of the accretion disk, $R_{\mathrm{in}}$, is set at the innermost stable circular orbit (ISCO) of the metric. Table 1 shows the best-fit values along with statistical uncertainties at the $90 \%$ confidence level. Figure 1 shows the data to the best-fit model ratio (middle panel). We know that the spin parameter and the deformation parameter have some degeneracy; we report this degeneracy between $a_{*}$ and $\alpha_{13}$ using a contour plot in Figure 2 (left panel).

Model $2 b$. Same as model $2 \mathrm{a}$ but with the location of the inner edge, $R_{\text {in }}$, free.

While it is a standard assumption to place the inner edge of the disk at the ISCO, in this model we leave $R_{\text {in }}$ free and fit for it. The best-fit values are reported in the fourth column in Table 1 . In particular, we find that $R_{\text {in }}$ prefers a value close to $R_{\text {ISCO }}$. This validates the assumption and for other models, we always set $R_{\text {in }}=R_{\text {ISCO }}$.
Model 2c. Same as model 2 a but with $q_{\text {out }}=3$.

We impose $q_{\text {out }}=3$, which is the value expected in the lamppost coronal geometry ${ }^{8}$ at large radii without light bending. The best-fit values are reported in the fifth column in Table 1 and the ratio of data to the best-fit model is shown in Figure 1 (bottom panel). The right panel in Figure 2 shows the degeneracy contours of $a_{*}$ versus $\alpha_{13}$. As we can see, the Kerr metric is not recovered in this case. This shows the importance of the emissivity profile in testing the Kerr metric. We discuss this point in more detail in Section 5.

Model 3. TBABS*RELXILL_NK with $\alpha_{22}$ as the deformation parameter.

With this model we constrain the deformation parameter $\alpha_{22}$. The assumptions are the same as in model $2 \mathrm{a}$, with $R_{\text {in }}=R_{\mathrm{ISCO}}$ and $q_{\text {out }}$ free. The best-fit values are reported in the sixth column in Table 1. Figure 3 shows the degeneracy contours of $a_{*}$ versus $\alpha_{22}$.

\section{Discussion}

We now discuss some aspects of the analysis presented above in particular and of the technique in general. To begin, our best-fit model (model $2 \mathrm{a}$ ) for $\alpha_{13}$ provides the constraints,

$$
a_{*}>0.975, \quad-0.34<\alpha_{13}<0.16,
$$

at the $99 \%$ confidence level. These constraints on $\alpha_{13}$ are the strongest ones obtained until now with our reflection model RELXILL_NK (see Cao et al. 2018, Tripathi et al. 2018, Wang-Ji et al. 2018, Bambi et al. 2018), and it is consistent with the Kerr metric. The strength of the present constraint can to be

\footnotetext{
8 This is a common model for the corona, see Dauser et al. (2013) for details.
} 

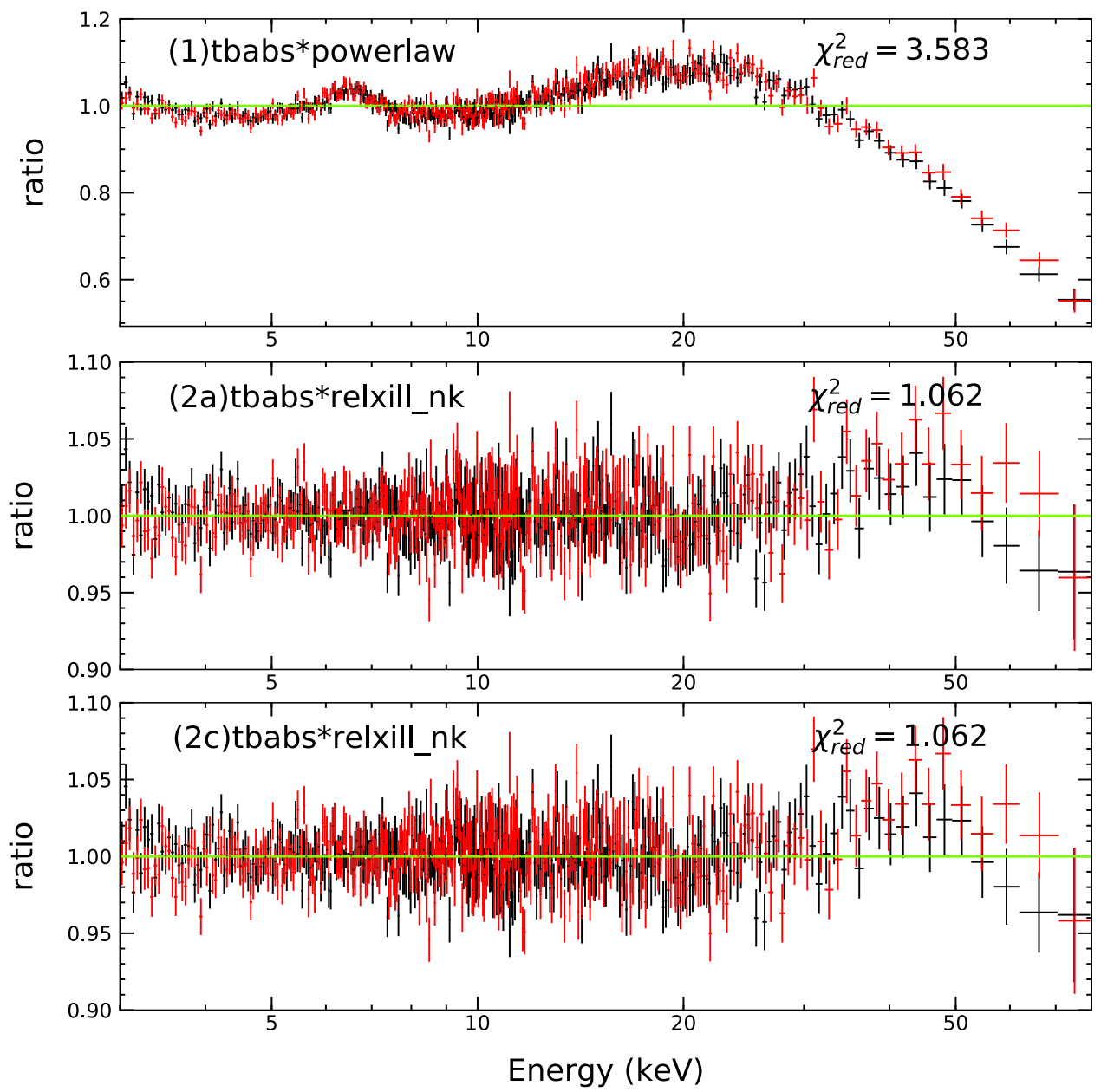

Figure 1. Data for the best-fit model ratio for model 1 (top panel), model 2a (middle panel), and model 2c (bottom panel). Black crosses are used for FPMA and red crosses are used for FPMB. See the text for more details.

attributed to the following factors. First, the source analyzed here is a black hole binary observed with NUSTAR, whereas in Cao et al. (2018) and Tripathi et al. (2018) the sources are supermassive black holes and therefore the observations there have a lower photon count. Second, in Wang-Ji et al. (2018), although the source is an X-ray binary, the spin of the hole is not as high as reported here and the inner edge of the disk (assumed to be colocated with the ISCO) in Wang-Ji et al. (2018) does not extend as close to the horizon as it does in the present case. The nature of the ISCO contours in the $a_{*}-\alpha_{13}$ phase space (see Johannsen 2013; Bambi et al. 2017) implies that the degeneracy between $a_{*}$ and $\alpha_{13}$ decreases as $a_{*}$ increases. Therefore, with a high spin estimate in the present case, we obtain stronger constraints on $\alpha_{13}$.

Our constraints on the second deformation parameter, $\alpha_{22}$, come from model 3,

$$
a_{*}>0.975, \quad-0.09<\alpha_{22}<0.42,
$$

at the $99 \%$ confidence level. These constraints on $\alpha_{22}$ are also stronger than previous constraints (Tripathi et al. 2018). Apart from the fact that the source in Tripathi et al. (2018) is a supermassive black hole, the best-fit spin is lower and the location of the ISCO is farther in that paper than reported here. Again, the nature of the ISCO contours in $a_{*}-\alpha_{22}$ phase space (see Johannsen 2013) imply that the degeneracy between $a_{*}$ and $\alpha_{22}$ decreases as $a_{*}$ increases. Therefore, a higher spin estimate in the present case provides better constraints on $\alpha_{22}$.

All the above results have accounted for the statistical uncertainty. Systematic uncertainties on the other hand are difficult to estimate and there is currently no detailed study in the literature to help to quantify them in the measurements employing reflection spectroscopy. We illustrate one aspect of systematic uncertainty with model $2 \mathrm{c}$. In this model, we fix the outer emissivity index, $q_{\text {out }}$, at three to mimic a specific coronal geometry, the lamppost. In the lamppost model, the corona is a point source located on the black hole spin axis at a few gravitational radii (more sophisticated geometries allow for an extended lamppost, a tube along the spin axis). For such a geometry, the emissivity index in the outer part of the accretion disk is equal to three. We report the best-fit parameters for model $2 \mathrm{c}$ in Table 1 and the data to the model ratio plot in Figure 1 (bottom panel). Nothing remarkable is apparent here: the reduced $\chi^{2}$ value for this model is comparable to other models, and the plot shows no obvious residuals (see the middle panel in Figure 1). The only outlier is the constraint on $\alpha_{13}$. Figure 2 in the right panel shows the confidence regions in the $a_{*}-\alpha_{13}$ phase space. The Kerr solution $\left(\alpha_{13}=0\right)$ is strongly violated. In the present case, since we artificially fix the parameter $q_{\text {out }}=3$, we can be confident that the exclusion of the Kerr solution is a systematic effect. Analysis with a version of RELXILL_NK that incorporates the lamppost 

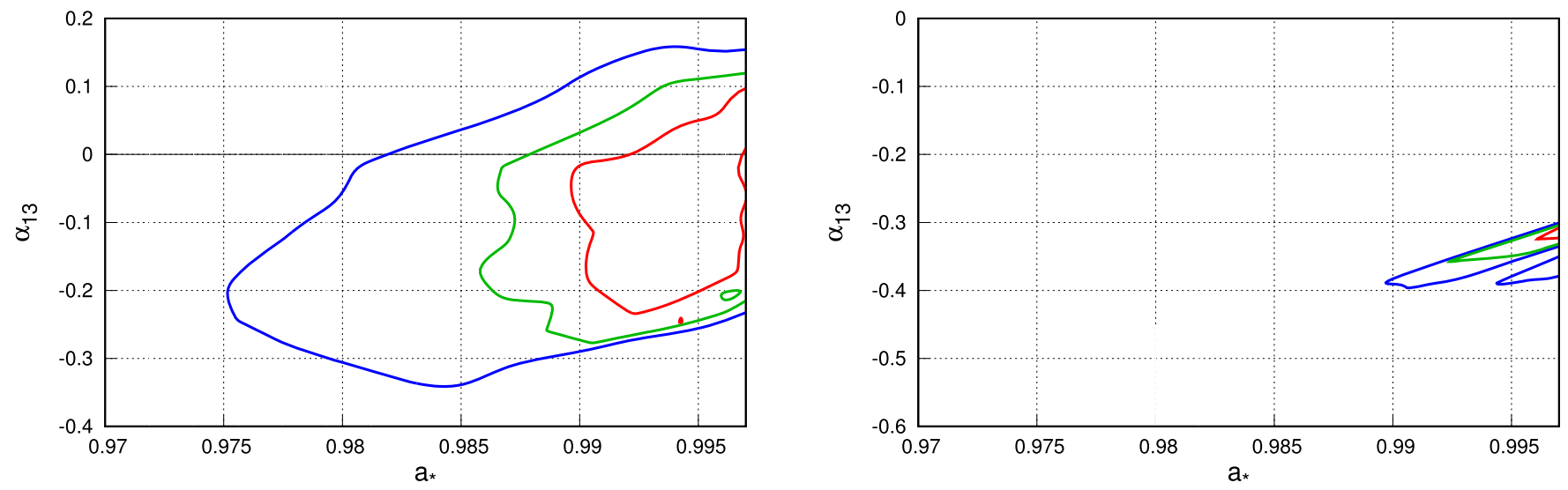

Figure 2. Constraints on the spin parameter $a_{*}$ and on the Johannsen deformation parameter $\alpha_{13}$ from model 2a (left panel) and model 2c (right panel). The red, green, and blue lines indicate, respectively, the $68 \%, 90 \%$, and $99 \%$ confidence level contours for two relevant parameters. The solid black line marks the Kerr solution.

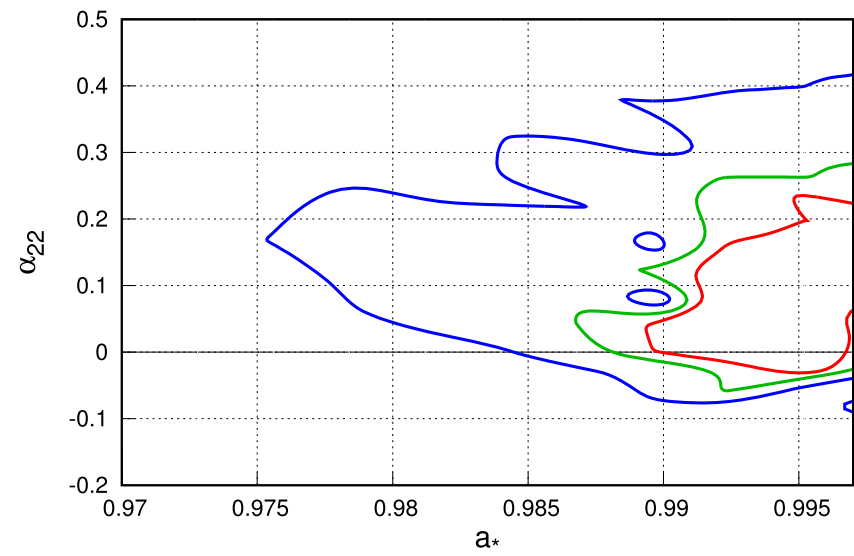

Figure 3. Constraints on the spin parameter $a_{*}$ and on the Johannsen deformation parameter $\alpha_{22}$ from model 3. The red, green, and blue lines indicate, respectively, the $68 \%, 90 \%$, and $99 \%$ confidence level contours for two relevant parameters. The solid black line marks the Kerr solution.

geometry will be better to judge whether such an exclusion indeed happens with the lamppost geometry with this source. Development of the lamppost version of RELXILL_NK is currently underway and we hope to resolve this conundrum in near future.

A second source of systematic uncertainty could be the assumption of a Novikov-Thorne-type thin disk in RELXILL_NK. This is thought to be correct with a good approximation for sources accreting between $5 \%$ and $30 \%$ of their Eddington limit (Penna et al. 2010; McClintock et al. 2014). In the case of GS 1354-645, the distance is poorly constrained and should be between 25 and $61 \mathrm{kpc}$ (Casares et al. 2009). For its mass, we have the lower bound $M \geqslant 7.6 \pm 0.7 M_{\odot}$ (Casares et al. 2009). The resulting luminosity is $L / L_{\mathrm{Edd}} \leqslant 0.53$, which is consistent with the 5\%-30\% range but it also allows higher and lower luminosities. For higher luminosities, the gas pressure may not be negligible and the inner edge of the disk may be at a radius smaller than the ISCO one, leading to an overestimate of the black hole spin.

\section{Concluding Remarks}

In a series of papers on precision tests of the Kerr solution with X-ray reflection spectroscopy we have developed a model for X-ray data analysis, namely RELXILL_NK, that incorporates parametrically deformed Kerr metrics in a disk-corona setup, and we have applied this model to several X-ray observations to obtain constraints on the deformation parameters which mark the departure from the Kerr solution. In the current study, we have applied RELXILL_NK (introduced in Bambi et al. 2017) to the X-ray binary GS $1354-645$. We report constraints on the deformation parameters $\alpha_{13}$ and $\alpha_{22}$ introduced in Johannsen (2013). Constraints obtained here are stronger than those reported previously in Cao et al. (2018), Tripathi et al. (2018), and Wang-Ji et al. (2018). We have discussed some aspects of the constraints on the deformation parameters and some systematic issues associated with this technique.

Precision tests of the Kerr geometry with X-ray reflection spectroscopy is a nascent field and significant scope for improvement exists. With new X-ray satellites planned for launch in near future (Zhang et al. 2016), we expect data of much higher quality. Improvements in RELXILL_NK, including other disk geometries, coronal geometries, and non-Kerr metrics are underway.

This work was supported by the National Natural Science Foundation of China (NSFC), grant No. U1531117, and Fudan University, grant No. IDH1512060. S.N. acknowledges support from the Excellence Initiative at Eberhard-Karls Universität Tübingen. A.B.A. also acknowledges the support from the Shanghai Government Scholarship (SGS). C.B. and J.A.G. also acknowledge support from the Alexander von Humboldt Foundation. J.J. is supported by the Cambridge Trust and the Chinese Scholarship Council Joint Scholarship Program (201604100032).

\section{ORCID iDs}

Cosimo Bambi (10 https://orcid.org/0000-0002-3180-9502

\section{References}

Abbott, B. P., Abbott, R., Abbott, T. D., et al. 2016, PhRvL, 116, 061102 Arnaud, K. A. 1996, in ASP Conf. Ser. 101, Astronomical Data Analysis Software and Systems V, ed. G. H. Jacoby \& J. Barnes (San Francisco, CA: ASP), 17

Bambi, C. 2012, PhRvD, 86, 123013

Bambi, C. 2013, PhRvD, 87, 023007

Bambi, C. 2017, RvMP, 89, 025001

Bambi, C. 2018, AnP, 530, 1700430

Bambi, C., Abdikamalov, A., Ayzenberg, D., et al. 2018, Univ, 4, 79 
Bambi, C., Cárdenas-Avendaño, A., Dauser, T., García, J. A., \& Nampalliwar, S. 2017, ApJ, 842, 76

Bambi, C., Jiang, J., \& Steiner, J. F. 2016, CQGra, 33, 064001

Barausse, E., \& Sotiriou, T. P. 2008, PhRvL, 101, 099001

Berti, E., Barausse, E., Cardoso, V., et al. 2015, CQGra, 32, 243001

Bertotti, B., Iess, L., \& Tortora, P. 2003, Natur, 425, 374

Brocksopp, C., Jonker, P. G., Fender, R. P., et al. 2001, MNRAS, 323, 517

Cao, Z., Nampalliwar, S., Bambi, C., Dauser, T., \& García, J. A. 2018, PhRvL, 120, 051101

Carter, B. 1971, PhRvL, 26, 331

Casares, J., Orosz, J. A., Zurita, C., et al. 2009, ApJS, 181, 238

Cunningham, C. T. 1975, ApJ, 202, 788

Dauser, T., Garcia, J., Wilms, J., et al. 2013, MNRAS, 430, 1694

Dickey, J. M., \& Lockman, F. J. 1990, ARA\&A, 28, 215

Dvali, G., \& Gomez, C. 2013, ForPh, 61, 742

El-Batal, A. M., Miller, J. M., Reynolds, M. T., et al. 2016, ApJL, 826, L12

Everitt, C. W. F., Debra, D. B., Parkinson, B. W., et al. 2011, PhRvL, 106, 221101

Frieman, J. A., Turner, M. S., \& Huterer, D. 2008, ARA\&A, 46, 385

García, J., Dauser, T., Lohfink, A., et al. 2014, ApJ, 782, 76

Giddings, S. B. 2014, PhRvD, 90, 124033

Giddings, S. B. 2017, NatAs, 1, 0067

Jiang, J., Bambi, C., \& Steiner, J. F. 2015, ApJ, 811, 130

Johannsen, T. 2013, PhRvD, 88, 044002
Johannsen, T., \& Psaltis, D. 2013, ApJ, 773, 57

Kramer, M., Stairs, I. H., Manchester, R. N., et al. 2006, Sci, 314, 97

Krawczynski, H. 2012, ApJ, 754, 133

Makino, F. 1987, IAUC, 4342, 1

Mathur, S. D. 2005, ForPh, 53, 793

McClintock, J. E., Narayan, R., \& Steiner, J. F. 2014, SSRv, 183, 295

Miller, J. M., Reynolds, M. T., \& Kennea, J. 2015, ATel, 7612

Penna, R. F., McKinney, J. C., Narayan, R., et al. 2010, MNRAS, 408, 752

Psaltis, D., Perrodin, D., Dienes, K. R., \& Mocioiu, I. 2008, PhRvL, 100, 119902

Robinson, D. C. 1975, PhRvL, 34, 905

Sahni, V. 2004, in The Physics of the Early Universe, ed. E. Papantonopoulos (Berlin: Springer), 141

Tripathi, A., Nampalliwar, S., Abdikamalov, A. B., et al. 2018, PhRvD, 98, 023018

Wang-Ji, J., Abdikamalov, A. B., Ayzenberg, D., et al. 2018, arXiv:1806. 00126

Williams, J. G., Turyshev, S. G., \& Boggs, D. H. 2004, PhRvL, 93, 261101

Wilms, J., Allen, A., \& McCray, R. 2000, ApJ, 542, 914

Yagi, K., \& Stein, L. C. 2016, CQGra, 33, 054001

Yunes, N., \& Siemens, X. 2013, LRR, 16, 9

Zhang, S. N., Feroci, M., Santangelo, A., et al. 2016, Proc. SPIE, 9905, 99051Q 